PROCEEDINGS OF THE

AMERICAN MATHEMATICAL SOCIETY

Volume 125, Number 2, February 1997, Pages 315-321

S $0002-9939(97) 03886-0$

\title{
PRODUCT OF DISTINCT SIMPLE INTEGRALLY CLOSED IDEALS IN 2-DIMENSIONAL REGULAR LOCAL RINGS
}

\author{
MEE-KYOUNG KIM
}

(Communicated by Eric M. Friedlander)

\begin{abstract}
Let $(R, m)$ be a two-dimensional regular local ring and $I$ an $m$ primary integrally closed ideal in $R$. In this paper, we give equivalent conditions for $I$ to be a product of distinct simple $m$-primary integrally closed ideals (i.e., $I=I_{1} \cdots I_{l}$, where $I_{1}, \cdots, I_{l}$ are distinct simple $m$-primary integrally closed ideals of $R$ ) in terms of the regularity of $R[I t] / p$ for all $p \in \operatorname{Min}(m R[I t])$ and in terms of how to choose a minimal generating set for $I$ over its minimal reductions.
\end{abstract}

\section{IntRoduction}

Throughout this paper, $(R, m, k)$ will denote a 2 -dimensional regular local ring with residue field $k$ and with quotient field $K$. Let $I$ be an $m$-primary integrally closed ideal in $(R, m, k)$. By Zariski's Unique Factorization Theorem, $I=$ $I_{1}^{\mu_{1}} \cdots I_{l}^{\mu_{l}}, \mu_{i} \geq 1$, where $I_{1}, \cdots, I_{l}$ are distinct simple $m$-primary integrally closed ideals in $(R, m, k)$. In [2], Huneke and Sally showed that if $I$ is a simple $m$-primary integrally closed ideal in $(R, m, k)$, then $R[I t] / p$ is regular, where $p=\operatorname{Min}(m R[I t])$. We extend this result to the case of an integrally closed ideal which is the product of distinct simple integrally closed ideals. To wit, we prove that $\mu_{i}=1$ for all $i=1, \cdots, l$ if and only if $R[I t] / p_{i}$ is regular for all $p_{i} \in \operatorname{Min}(m R[I t])$. Let $v_{i}$ be the valuation of $R[I t]_{p_{i}} \cap K$ for all $p_{i} \in \operatorname{Min}(m R[I t])$. We shall show that the following conditions are equivalent:

(1) $R[I t] / p_{i}$ is regular for all $p_{i} \in \operatorname{Min}(m R[I t])$.

(2) For any reduction $(a, b)$ of $I$, there exist elements $c_{i_{1}}, \cdots, c_{i_{n}}$ in $I$ such that $a, b, c_{i_{1}}, \cdots, c_{i_{n}}$ is a minimal generating set of $I$ and $v_{i}\left(c_{i_{j}}\right)>v_{i}(a)=v_{i}(b)$ for $i=1, \cdots, l$ and $j=1, \cdots, n$.

(3) There exist a reduction $(a, b)$ of $I$ and elements $c_{i_{1}}, \cdots, c_{i_{n}}$ in $I$ such that $a, b, c_{i_{1}}, \cdots, c_{i_{n}}$ is a minimal generating set of $I$ and $v_{i}\left(c_{i_{j}}\right)>v_{i}(a)=v_{i}(b)$ for $i=1, \cdots, l$ and $j=1, \cdots, n$.

This paper is divided into three sections. Section 2 deals with some preliminary facts. In section 3, we shall prove our main theorems.

\section{Preliminaries}

Throughout this paper, all rings are assumed to be commutative with identity. By a local ring $(A, n)$, we mean a Noetherian ring $A$ which has a unique maximal

Received by the editors April 28, 1993.

1991 Mathematics Subject Classification. Primary 13H05. 
ideal $n$. By $\operatorname{dim}(A)$ we always mean the Krull dimension of $A$. A regular local ring $(A, n)$ is a local ring whose maximal ideal can be generated by $d=\overline{\operatorname{dim}(A) \text { elements. }}$ We will use the abbreviation RLR for regular local ring. Recall that an ideal is simple if it is not the unit ideal and has no nontrivial factorization. An element $\overline{a \in A}$ is said to be integral over an ideal $I$ of $A$ if $a$ satisfies an equation of the form

$$
a^{n}+r_{1} a^{n-1}+\cdots+r_{n}=0, \quad r_{i} \in I^{i} .
$$

The set of all elements in $A$ which are integral over an ideal $I$ forms an ideal, denoted by $\bar{I}$ and called the integral closure of $I$. An ideal $I$ is said to be integrally closed (or equivalently "complete") if $I=\bar{I}$.

Let $(A, n)$ be a local ring and $I$ an ideal of $A$. An ideal $J$ contained in $I$ is called a reduction of $I$ if $J I^{s}=I^{s+1}$ for some integer $s \geq 0$. A reduction $J$ of $I$ is called a minimal reduction of $I$ if $J$ is minimal with respect to being a reduction of $I$. D. Northcott and D. Rees proved that if $A / n$ is infinite, then any minimal generating set for a minimal reduction of an ideal $I$ is analytically independent ([6, Lemma 2, p. 149]). We will use the notation $\lambda_{A}(M)$ (or simply $\lambda(M)$ ) to denote the length of $M$ as an $A$-module, $\mu(I)$ to denote the number of elements in a minimal basis of an ideal $I$ in $A$ (i.e., $\mu(I)=\lambda(I / n I)$ ), and $e(A)$ to denote the multiplicity of the maximal ideal $n$ of $A$. The order $o(I)$ of an ideal $I$ of a local ring $(A, n)$ is $r$ if $I \subseteq n^{r}$ but $I \nsubseteq n^{r+1}$. We refer the reader to [4], [1], [2] , or [7] for any unexplained notation or terminology.

Let $A$ be a Noetherian domain with quotient field $L$, and let $I$ be an ideal of $A$. Let $\overline{A\left[I t, t^{-1}\right]}$ be the integral closure of $A\left[I t, t^{-1}\right]$ in the quotient field $L(t)$, and let $\left\{Q_{1}, \cdots, Q_{n}\right\}$ be all the minimal primes of $\left(t^{-1}\right) \overline{A\left[I t, t^{-1}\right]}$. Then $\overline{A\left[I t, t^{-1}\right]}$ is a Krull domain and each $Q_{i}$ is a height one prime, and hence $\left(\overline{A\left[I t, t^{-1}\right]}\right)_{Q_{i}}$ is a discrete valuation ring of $L(t)$ for $i=1, \cdots, n$. The Rees valuation rings of $I$ are

$$
\left\{\left(\overline{A\left[I t, t^{-1}\right]}\right)_{Q} \cap L \mid Q \in \operatorname{Min}\left(\left(t^{-1}\right) \overline{A\left[I t, t^{-1}\right]}\right)\right\} .
$$

The set of corresponding discrete valuations of these Rees valuation rings of $I$ are called the set of Rees valuations of $I$ and denoted by $T(I)$, i.e.,

$$
\begin{gathered}
T(I)=\left\{v \mid v \text { is the valuation of }\left(\overline{A\left[I t, t^{-1}\right]}\right)_{Q} \cap L,\right. \\
\left.Q \in \operatorname{Min}\left(\left(t^{-1}\right) \overline{A\left[I t, t^{-1}\right]}\right)\right\} .
\end{gathered}
$$

In a $d$-dimensional local domain $(A, n, l)$ with quotient field $L$, by a prime divisor of the second kind on $A$ (or equivalently prime divisor of $(A, n)$ ) we mean a discrete valuation $v$ of $L$ on $A$ which is non-negative on $A$ and has center $n$ on $A$ and whose residual transcendence degree (denoted by $\left.\operatorname{tr} \cdot \operatorname{deg}_{l} k(v)\right)$ is $d-1$. We denote the residue field of the valuation ring of the valuation $v$ by $k(v)$.

Concerning the structure of the integrally closed ideal in a 2-dimensional RLR $(R, m, k)$, O. Zariski proved the following beautiful theorems which are the main background for this paper.

Theorem 2.1. (Zariski's Unique Factorization Theorem: [7, Appendix 5, Theorem 3]). Every integrally closed ideal of $(R, m, k)$ can be uniquely factored into the product of simple integrally closed ideals (up to order). 
Theorem 2.2 (Zariski's Product Theorem: [7], Appendix 5, Theorem 2). The product of integrally closed ideals in $(R, m, k)$ is again integrally closed.

Theorem 2.3. (Zariski's One-to-One Correspondence Theorem: [7, Appendix 5, Theorem E]). The simple m-primary integrally closed ideals of $(R, m, k)$ are in one-to-one correspondence with the prime divisors of the second kind on $(R, m, k)$.

Let $I$ be an $m$-primary integrally closed ideal in $(R, m, k)$. Then all the powers of $I$ are integrally closed by Zariski's Product Theorem, hence the set of Rees valuations of $I$ is

$$
\begin{aligned}
T(I)= & \left\{v \mid v \text { is the valuation of }\left(R\left[I t, t^{-1}\right]\right)_{Q} \cap K,\right. \\
& \left.Q \in \operatorname{Min}\left(\left(t^{-1}\right) R\left[I t, t^{-1}\right]\right)\right\} \\
= & \left\{v \mid v \text { is the valuation of }(R[I t])_{p} \cap K, p \in \operatorname{Min}(m R[I t])\right\} \\
= & \bigcup_{\substack{a \neq 0 \\
a \in I}}\left\{v \mid v \text { is the valuation of }(R[I / a])_{q}, q \in \operatorname{Min}(a R[I / a])\right\} .
\end{aligned}
$$

By Zariski's Unique Factorization Theorem, $I=I_{1}^{\mu_{1}} \cdots I_{l}^{\mu_{l}}, \mu_{i} \geq 1$, where $I_{1}, \cdots, I_{l}$ are distinct simple $m$-primary integrally closed ideals in $R$. By Zariski's One-toOne Correspondence Theorem, if $\operatorname{Min}(m R[I t])=\left\{p_{1}, \cdots, p_{r}\right\}$, then $r=l$ and upon reordering, $v_{i}$ is the discrete valuation of $R[I t]_{p_{i}} \cap K$, where $v_{i}$ is the prime divisor associated to $I_{i}$ for $i=1, \cdots, l$.

Remarks 2.4. (1) Let $I$ be an $m$-primary integrally closed ideal in $(R, m, k)$, and let $p$ be any minimal prime of $m R[I t]$. Then $p$ is a homogeneous prime ideal of $h t(p)=1$. Since $N=(m, I t)$ is the unique maximal homogeneous ideal in $R[I t]$ and $p \supseteq m R[I t]$, the piece of degree 0 in $p$ is $m$. Hence $R[I t] / p$ is a 2-dimensional graded domain over the field $k$.

(2) Let $k$ be a field and $A=k\left[a_{1}, \cdots, a_{d}\right]$, where $a_{1}, \cdots, a_{d} \in A$. Define the canonical homomorphism $\phi$ from $k\left[X_{1}, \cdots, X_{d}\right]$ onto $A$ with $\phi\left(X_{i}\right)=a_{i}$ for $i=$ $1, \cdots, d$ where $X_{1}, \cdots, X_{d}$ are variables. If $\operatorname{dim}(A)=d \geq 1$, then $A$ is a polynomial ring in $d$-variables over $k$, since the kernel of $\phi$ is zero.

Lemma 2.5. Let $G=k\left[G_{1}\right]=G_{0} \oplus G_{1} \oplus G_{2} \oplus \cdots$, where $G_{0}=k$, be a $d$ dimensional graded homogeneous Noetherian domain over a field $k, d \geq 1$. Then $G$ is regular if and only if $G$ is a polynomial ring in $d$-variables over the field $k$.

Proof. $(\Leftarrow)$ : This is clear.

$(\Rightarrow)$ : Suppose that $G$ is regular. Let $N=G_{1} G$ be the unique homogeneous maximal ideal in $G$. Hence $G_{N}$ is a $d$-dimensional RLR since $h t(N)=d$. We can choose elements $a_{1}, \cdots, a_{d}$ in $G_{1}$ such that $N G_{N}=\left(a_{1}, \cdots, a_{d}\right) G_{N}$. Let $\mu(N)=n \geq d$. Then we can choose elements $a_{d+1}, \cdots, a_{n}$ in $G_{1}$ such that $N=\left(a_{1}, \cdots, a_{d}, a_{d+1}, \cdots, a_{n}\right) G$.

Claim. $N=\left(a_{1}, \cdots, a_{d}\right) G$.

$\supseteq$ : This is clear.

$\subseteq:$ For $i=d+1, \cdots, n$, we can express

$$
a_{i}=a_{1} \cdot\left(g_{i_{1}} / \alpha_{i_{1}}\right)+\cdots+a_{d} \cdot\left(g_{i_{d}} / \alpha_{i_{d}}\right),
$$

where $g_{i_{1}}, \cdots, g_{i_{d}} \in G$ and $\alpha_{i_{1}}, \cdots, \alpha_{i_{d}} \notin N$. Express $g_{i_{j}}=u_{i_{j}}+h_{i_{j}}$, where $u_{i_{j}} \in k$ and $h_{i_{j}} \in N$ for $j=1, \cdots, d$. Considering the degree of each term, $h_{i_{j}}=0$ for $j=1, \cdots, d$. Hence we have $a_{i} \in\left(a_{1}, \cdots, a_{d}\right) k \subseteq\left(a_{1}, \cdots, a_{d}\right) G$ for $i=d+1, \cdots, n$. 
Therefore $G=k\left[a_{1}, \cdots, a_{d}\right]$ is a polynomial ring in $d$-variables over $k$ by Remarks $2.4(2)$.

\section{FACTORIZATION OF AN INTEGRALLY CLOSED IDEAL}

Theorem 3.1. Let $I$ be an m-primary integrally closed ideal in $(R, m, k)$. Assume that $\operatorname{Min}(m R[I t])=\left\{p_{1}, \cdots, p_{l}\right\}$ and that $k$ is an algebraically closed field. Then the following conditions are equivalent:

(1) $R[I t] / p_{i}$ is regular for $i=1, \cdots, l$.

(2) $I=I_{1} \cdots I_{l}$, where $I_{1}, \cdots, I_{l}$ are distinct simple m-primary integrally closed ideals in $R$.

Proof. Let $T(I)=\left\{v_{1}, \cdots, v_{l}\right\}$ be the Rees valuations of $I$. Then, by Theorem 2.1 and Theorem 2.3, we can write $I=I_{1}^{\mu_{1}} \cdots I_{l}^{\mu_{l}}$, where $I_{i}$ is a simple $m$-primary integrally closed ideal in $R$ associated to $v_{i}$ for $i=1, \cdots, l$.

Let $S=R[I t]$ and $N=(m, I t) S$ be the unique maximal homogeneous ideal in $S$. Then $I / m I \oplus I^{2} / m I^{2} \oplus \cdots$, denoted by $\bar{N}$, is the unique maximal homogeneous ideal in $S / m S$. Let $T=(S / m S)_{\bar{N}}$. By Corollary 3.4 in [2] and Corollary 3.2 in [1], we have

$$
e(T)=o(I)
$$

Since $\operatorname{Min}(m S)=\left\{p_{1}, \cdots, p_{l}\right\}$ and $N$ is the unique maximal homogeneous ideal in $S, \operatorname{Min}(S / m S)=\left\{\bar{p}_{1}, \cdots, \bar{p}_{l}\right\}$, where $\bar{p}_{i}$ denotes the image of $p_{i}$ in $S / m S$ and $\operatorname{dim}\left(\left(S / p_{i}\right)_{N}\right)=2$ for $i=1, \cdots, l$. Hence the associativity formula ([4, Theorem 14.7]) gives the following:

$$
e(T)=\sum_{i=1}^{l} e\left(\left(N / p_{i}\right)_{N},\left(S / p_{i}\right)_{N}\right) \cdot \lambda\left((S / m S)_{p_{i}}\right)
$$

$(1) \Rightarrow(2)$ : Suppose that $S / p_{i}$ is regular for $i=1, \cdots, l$. From $(* *)$, we have

$$
\begin{aligned}
e(T) & =\sum_{i=1}^{l} \lambda\left((S / m S)_{p_{i}}\right) \\
& =\sum_{i=1}^{l} v_{i}(m) \\
& =\sum_{i=1}^{l} o\left(I_{i}\right) \quad([3], \text { Proposition 21.4) } \\
& =o\left(I_{1} \cdots I_{l}\right) \\
& \leq o(I) .
\end{aligned}
$$

From $(*)$, we have $o(I)=o\left(I_{1} \cdots I_{l}\right)$. This implies that $I=I_{1} \cdots I_{l}$ since $I_{1} \cdots I_{l}$ divides $I$.

$(2) \Rightarrow(1)$ : Suppose that $I=I_{1} \cdots I_{l}$, where $I_{1}, \cdots, I_{l}$ are distinct simple $m$ primary integrally closed ideals in $R$. By Proposition 21.4 in [3], we have

$$
o(I)=\sum_{i=1}^{l} o\left(I_{i}\right)=\sum_{i=1}^{l} v_{i}(m)
$$


Using $(*)$ and $(* *)$, we have

$$
\sum_{i=1}^{l} v_{i}(m)=\sum_{i=1}^{l} e\left(\left(S / p_{i}\right)_{N}\right) \cdot v_{i}(m) .
$$

Hence

$$
\sum_{i=1}^{l} v_{i}(m)\left[e\left(\left(S / p_{i}\right)_{N}\right)-1\right]=0 .
$$

Since $v_{i}(m)>0$ and $e\left(\left(S / p_{i}\right)_{N}\right) \geq 1$ for each $i=1, \cdots, l$, we have $e\left(\left(S / p_{i}\right)_{N}\right)=1$ for $i=1, \cdots, l$. $S$ is a Cohen-Macaulay ring by Theorem 3.2 in [2], and hence $\left(S / p_{i}\right)_{N}$ is unmixed for $i=1, \cdots, l$ which implies that $\left(S / p_{i}\right)_{N}$ is a RLR for $i=1, \cdots, l\left(\left[5\right.\right.$, Theorem 40.6]). Therefore $S / p_{i}$ is regular for $i=1, \cdots, l$.

Corollary 3.2 ([2], Theorem 3.8). Assume that $k$ is an algebraically closed field. Let $I$ be a simple $m$-primary integrally closed ideal in $(R, m, k)$. If $\operatorname{Min}(m R[I t])=$ $\{p\}$, then $R[I t] / p$ is regular.

We remark that the statement of Theorem 3.1 is not true in general if the product of distinct simple ideals is replaced by the product of powers of simple ideals. For example, let $R=k[x, y]_{(x, y)}$ with an algebraically closed field $k$ and $m=(x, y) R$. Let $I=m^{2}$. Then the set of Rees valuations of $I$ is $\{o\}$, where $o$ is the order valuation of $R$. In $R[m t]$, we have $o\left(t^{-1}\right)=o(m)=1$. Since $R\left[m^{2} t^{2}\right]$ is a subring of $R[m t], o\left(t^{2}\right)=-2$ in $R\left[m^{2} t^{2}\right]$. Since $R\left[m^{2} t\right]$ is isomorphic to $R\left[m^{2} t^{2}\right], o(t)=-2$ in $R\left[m^{2} t\right]$. In $R\left[m^{2} t\right], o\left(x^{2} t\right)=o(x y t)=o\left(y^{2} t\right)=0$. Hence $x^{2} t, x y t$ and $y^{2} t$ are not in $p$, where $\{p\}=\operatorname{Min}\left(m R\left[m^{2} t\right]\right)$. So we have $R\left[m^{2} t\right] / p \cong k[X, Y, Z] /\left(Z^{2}-X Y\right)$, where $X, Y, Z$ are variables, which is a 2-dimensional normal domain, but not regular.

Without the assumption that the residue field $k$ is algebraically closed, we give in Theorem 3.3 some equivalent conditions in order that $R[I t] / p_{i}$ be regular for $i=1, \cdots, l$, which deal with the generating set for $I$ over a minimal reduction.

Theorem 3.3. Let $I$ be an m-primary integrally closed ideal in $(R, m, k)$. Assume that $\operatorname{Min}(m R[I t])=\left\{p_{1}, \cdots, p_{l}\right\}$. Let $v_{i}$ be the valuation of $R[I t]_{p_{i}} \cap K$ for $i=$ $1, \cdots, l$. Then the following conditions are equivalent:

(1) $R[I t] / p_{i}$ is regular for $i=1, \cdots, l$.

(2) For any reduction $(a, b)$ of $I$, there exist elements $c_{i_{1}}, \cdots, c_{i_{n}}$ in $I$ such that $a, b, c_{i_{1}}, \cdots, c_{i_{n}}$ is a minimal generating set of $I$ and $v_{i}\left(c_{i_{j}}\right)>v_{i}(a)=v_{i}(b)$ for $i=1, \cdots, l$ and $j=1, \cdots, n$.

(3) There exist a reduction $(a, b)$ of $I$ and elements $c_{i_{1}}, \cdots, c_{i_{n}}$ in $I$ such that $a, b, c_{i_{1}}, \cdots, c_{i_{n}}$ is a minimal generating set of $I$ and $v_{i}\left(c_{i_{j}}\right)>v_{i}(a)=v_{i}(b)$ for $i=1, \cdots, l$ and $j=1, \cdots, n$.

Proof. $(2) \Rightarrow(3)$ : This is clear.

$(3) \Rightarrow(1)$ : Let $J_{i}=\left(m, c_{i_{1}} t, \cdots, c_{i_{n}} t\right)$ for $i=1, \cdots, l$ be a homogeneous ideal in $R[I t]$. Since $v_{i}\left(c_{i_{j}}\right)>v_{i}(a)=v_{i}(b)$ for $j=1, \cdots, n$, we have $v_{i}\left(c_{i_{j}} t\right)>v_{i}(a t)=0$. Hence $c_{i_{j}} t \in p_{i}$ for $j=1, \cdots, n$. Therefore we have $J_{i} \subseteq p_{i}$ for $i=1, \cdots, l$. Since $a, b, c_{i_{1}}, \cdots, c_{i_{n}}$ is a minimal generating set of $I$ for $i=1, \cdots, l$, we have

$$
R[I t] / J_{i} \cong(R / m)[\overline{a t}, \overline{b t}]
$$


where $\overline{a t}, \overline{b t}$ denote the homomorphic image of at, bt in $R[I t] / J_{i}$. And

$$
\operatorname{dim}\left(R[I t] / J_{i}\right) \geq \operatorname{dim}\left(R[I t] / p_{i}\right)=2 \quad \text { for } \quad i=1, \cdots, l .
$$

By Remarks 2.4(2), $R[I t] / J_{i}$ is a polynomial ring over $k$, and hence it is a domain for $i=1, \cdots, l$. This implies that $J_{i}=p_{i}$ for $i=1, \cdots, l$. Moreover, $R[I t] / p_{i}$ is regular for $i=1, \cdots, l$.

$(1) \Rightarrow(2)$ : Suppose that $R[I t] / p_{i}$ is regular for $i=1, \cdots, l$. By Remarks 2.4(1) and Lemma 2.5, we have $R[I t] / p_{i} \cong k[X, Y]$, where $X$ and $Y$ are variables over $k$. Let $J=(a, b)$ be any minimal reduction of $I$. Since $a, b$ are analytically independent, $m R[J t]$ is a homogeneous prime ideal in $R[J t]$, and hence $m R[I t] \cap$ $R[J t]=m R[J t]$. Moreover, we have

$$
\operatorname{dim}\left(R[J t] / p_{i} \cap R[J t]\right)=\operatorname{dim}\left(R[I t] / p_{i}\right)=\operatorname{dim}(R[J t] / m R[J t])=2 .
$$

Since $p_{i} \cap R[J t]$ and $m R[J t]$ are homogeneous prime ideals such that $p_{i} \cap R[J t] \supseteq$ $m R[J t]$, we obtain $p_{i} \cap R[J t]=m R[J t]$ for $i=1, \cdots, l$. Hence we have

$$
R[J t] / p_{i} \cap R[J t] \cong(R / m)[\bar{a} t, \bar{b} t]
$$

where $\bar{a} t, \bar{b} t$ denote the images of $a, b$ in $I / m I$. Hence we have the following map of homogeneous graded rings:

$$
\phi:(R / m)[\bar{a} t, \bar{b} t] \hookrightarrow R[I t] / p_{i} \quad(\cong k[X, Y])
$$

which is integral. Then $\bar{a} t, \bar{b} t$ are mapped by $\phi$ to homogeneous polynomials of degree 1 in $k[X, Y]$. Let $\phi(\bar{a} t)=\alpha X+\beta Y$ and $\phi(\bar{b} t)=u X+v Y$, where $\alpha, \beta, u, v \in$ $k$. It is not difficult to see that $\alpha X+\beta Y$ and $u X+v Y$ are linearly independent over $k$. By a suitable $k$-automorphism, we may assume that $\bar{a} t$ goes to $X$ and $\bar{b} t$ goes to $Y$. It is clear that $k[\bar{a} t, \bar{b} t]=k[X, Y]$. Let $\mu(I)=n+2$. We can extend $(a, b)$ to a minimal generating set $a, b, d_{1}, \cdots, d_{n}$ of $I$. For each $i=1, \cdots, l$, let

$$
\psi_{i}: R\left[a t, b t, d_{1} t, \cdots, d_{n} t\right] \longrightarrow R[I t] / p_{i} \quad(\cong k[X, Y])
$$

be a canonical map with $\psi_{i}(a t)=X$ and $\psi_{i}(b t)=Y$. Suppose that $\psi_{i}\left(d_{j} t\right)=$ $\overline{s_{i_{j}}} X+\overline{r_{i_{j}}} Y$, where $\overline{s_{i_{j}}}$ and $\overline{r_{i_{j}}}$ are in $k$ for $j=1, \cdots, n$. Let $s_{i_{j}}$ and $r_{i_{j}}$ be the preimages of $\overline{s_{i_{j}}}$ and $\overline{r_{i_{j}}}$ in $R$. Replace $c_{i_{j}}$ by $d_{j}-s_{i_{j}} a-r_{i_{j}} b$ for $j=1, \cdots, n$. Then $\left(a, b, c_{i_{1}}, \cdots, c_{i_{n}}\right)=\left(a, b, d_{1}, \cdots, d_{n}\right)$ is still a minimal generating set of $I$. Moreover, $\psi_{i}\left(c_{i_{j}} t\right)=0$ for $j=1, \cdots, n$. Hence $c_{i_{j}} t \in p_{i}$ for $j=1, \cdots, n$. Since $v_{i}$ is the discrete valuation of $R[I t]_{p_{i}} \cap K$ for $i=1, \cdots, l$, we have $v_{i}\left(c_{i_{j}} t\right)>0$ for $j=1, \cdots, n$. Hence we have $v_{i}\left(c_{i_{j}}\right)>v_{i}\left(t^{-1}\right)=v_{i}(I)$ for $j=1, \cdots, n$ and $i=1, \cdots, l$. Finally, for each $i=1, \cdots, l$, we have $v_{i}(I)=v_{i}(J)=v_{i}(a)=v_{i}(b)$, since $J=(a, b)$ is a reduction of $I$. The proof is complete.

Corollary 3.4. Let $I,(R, m, k),\left\{p_{1}, \cdots, p_{l}\right\}$, and $\left\{v_{1}, \cdots, v_{l}\right\}$ be as in Theorem 3.3. Further, assume that $k$ is algebraically closed. Then the following conditions are equivalent:

(1) $I=I_{1} \cdots I_{l}$, where $I_{1}, \cdots, I_{l}$ are distinct simple $m$-primary integrally closed ideals in $R$.

(2) For any reduction $(a, b)$ of $I$ and for all $d \in I$, there exist $r_{i}, s_{i} \in R$ such that $v_{i}\left(d-r_{i} a-s_{i} b\right)>v_{i}(I)$ for $i=1, \cdots, l$.

(3) There exists a reduction $(a, b)$ of $I$ such that for all $d \in I$, there exist $r_{i}, s_{i} \in R$ such that $v_{i}\left(d-r_{i} a-s_{i} b\right)>v_{i}(I)$ for $i=1, \cdots, l$. 
Proof. $(2) \Rightarrow(3)$ : This is clear.

$(3) \Rightarrow(1)$ : Assume that (3) is true. Then for each $i=1, \cdots, l$, we can extend $(a, b)$ to a minimal generating set $a, b, d_{i_{1}}, \cdots, d_{i_{n}}$ of $I$ such that

$$
v_{i}\left(d_{i_{j}}-r_{i_{j}} a-s_{i_{j}} b\right)>v_{i}(I),
$$

where $r_{i_{j}}$ and $s_{i_{j}}$ are in $R$ for $j=1, \cdots, n$. Replace $d_{i_{j}}$ by $c_{i_{j}}=d_{i_{j}}-r_{i_{j}} a-s_{i_{j}} b$ for $j=1, \cdots, n$. Then $a, b, c_{i_{1}}, \cdots, c_{i_{n}}$ is still a minimal generating set of $I$, and $v_{i}\left(c_{i_{j}}\right)>v_{i}(a)=v_{i}(b)$, since $(a, b)$ is a reduction of $I$. By Theorem $3.3, I=I_{1} \cdots I_{l}$, where $I_{1}, \cdots, I_{l}$ are distinct simple $m$-primary integrally closed ideals in $R$.

$(1) \Rightarrow(2)$ : Assume that (1) is true. By Theorem 3.3, for any reduction $(a, b)$ of $I$, there exist elements $c_{i_{1}}, \cdots, c_{i_{n}}$ in $I$ such that $a, b, c_{i_{1}}, \cdots, c_{i_{n}}$ is a minimal generating set of $I$ and $v_{i}\left(c_{i_{j}}\right)>v_{i}(a)=v_{i}(b)$ for $i=1, \cdots, l$ and $j=1, \cdots, n$. Let $d$ be an arbitrary element in $I$. If $d \in\left\{a, b, c_{i_{1}}, \cdots, c_{i_{n}}\right\}$, then it is not difficult to see the required form. If $d \in I \backslash\left\{a, b, c_{i_{1}}, \cdots, c_{i_{n}}\right\}$, then we can express $d=$ $r_{i} a+s_{i} b+\sum_{j=1}^{n} \alpha_{i_{j}} c_{i_{j}}$, where $r_{i}, s_{i}$ and $\alpha_{i_{j}}$ are in $R$ for $j=1, \cdots, n$ and $i=1, \cdots, l$. Hence we have

$$
\begin{aligned}
v_{i}\left(d-r_{i} a-s_{i} b\right) & =v_{i}\left(\sum_{j=1}^{n} \alpha_{i_{j}} c_{i_{j}}\right) \\
& \geq \min _{j=1, \cdots, n}\left\{v_{i}\left(\alpha_{i_{j}} c_{i_{j}}\right)\right\} \\
& \geq \min _{j=1, \cdots, n}\left\{v_{i}\left(c_{i_{j}}\right)\right\} \\
& >v_{i}(a) \\
& =v_{i}(I) .
\end{aligned}
$$

Corollary 3.5 ([2], Lemma 3.7). Let $v$ be a prime divisor of $R$ with associated simple $m$-primary integrally closed ideal $I$ in $(R, m, k)$. Suppose that $k$ is algebraically closed. Then there exists a reduction $(a, b)$ of I such that for all $d \in I$, there exist $s$ and $r$ in $R$ such that $v(d-s a-r b)>v(I)$.

\section{REFERENCES}

1. C. Huneke, Complete ideals in two-dimensional regular local rings, Microprogram in Commutative Algebra, MSRI, Springer-Verlag (1987).

2. C. Huneke and J. Sally, Birational extensions in dimension two and integrally closed ideals, J. of Algebra 115 (2) (1988), 481-500. MR 89e:13025

3. J. Lipman, Rational singularities, with applications to algebraic surfaces and unique factorization, Inst. Hautes Études Sci. Publ. Math. 36 (1969), 195-279. MR 43:1986

4. H. Matsumura, Commutative ring theory, Cambridge Studies in Advanced Math. 8, Cambridge Univ. Press, Cambridge, 1986. MR 88h:13001

5. M. Nagata, Local Rings, Interscience, New York, 1962. MR 27:5790

6. D. G. Northcott and D. Rees, Reductions of ideals in local rings, Proc. Cambridge Phil. Soc. 50 (1954), 145-158. MR 15:596a

7. O. Zariski and P. Samuel, Commutative Algebra Vol.II, Von Nostrand, Princeton, 1960. MR 22:11006

Department of Mathematics, Sung Kyun Kwan University, Suwon 440-746, Korea

E-mail address: mkkim@yurim.skku.ac.kr 